\title{
SEMI-PRIME NOETHERIAN RINGS OF INJECTIVE DIMENSION ONE
}

\author{
A. W. CHATTERS \\ School of Mathematics, University of Bristol, University Walk, Bristol BS8 1TW, UK \\ e-mail: arthur.chatters@bristol.ac.uk
}

(Received 10 September, 2004; accepted 17 January, 2005)

\begin{abstract}
Let $R$ be a semi-prime Noetherian ring of injective dimension 1. Let $P$ be a minimal prime ideal of $R$. In this paper it is shown that $R / P$ need not have injective dimension 1 . Necessary and sufficient conditions are given for $R / P$ to have injective dimension 1 .
\end{abstract}

2000 Mathematics Subject Classification. 16P40.

1. Introduction. Let $R$ be a semi-prime Noetherian ring of injective dimension 1 . Because hereditary rings have injective dimension 1 , it is reasonable to hope that results in the hereditary case may suggest properties which can be proved about the more general ring $R$. It was shown by Levy [3, Theorem 4.3] that in the hereditary case $R$ is a direct sum of prime rings, but this does not generalise in a straightforward way because the integral group ring $\mathbb{Z}[G]$ of any non-trivial finite group $G$ is a semiprime Noetherian ring of injective dimension 1 but it is not a direct sum of prime rings. Thus, if $P$ is a minimal prime ideal of $R$, we cannot expect $R / P$ to be a direct summand of $R$, but we might at least hope that $R / P$ has injective dimension 1 . The two purposes of this paper are firstly to show that $R / P$ need not have injective dimension 1 (Example 2.1) and then to give a necessary and sufficient condition for $R / P$ to have injective dimension 1 . The condition is that $(I+P) / P$ is an invertible ideal of $R / P$, where $I$ is the intersection of all the minimal prime ideals of $R$ except $P$ (Corollary 3.11).

2. The example. It is well known that if $G$ is any finite group then the integral group ring $\mathbb{Z}[G]$ is a semi-prime Noetherian ring of injective dimension 1 . Using $A_{4}$ as usual to denote the alternating group on four symbols, we shall show that $\mathbb{Z}\left[A_{4}\right]$ has a prime factor ring which does not have injective dimension 1 (we believe that $A_{4}$ is the smallest group with this property).

EXAMPLE 2.1. Set $S=M_{3}(\mathbb{Z})$. We start by constructing a specific representation of $A_{4}$ inside $S$. Let $H$ consist of all elements of $S$ of the three following types, where in each case $a, b, c$ are any integers with $a b c=1$ :

$$
\left(\begin{array}{lll}
a & 0 & 0 \\
0 & b & 0 \\
0 & 0 & c
\end{array}\right) ; \quad\left(\begin{array}{lll}
0 & a & 0 \\
0 & 0 & b \\
c & 0 & 0
\end{array}\right) ; \quad\left(\begin{array}{lll}
0 & 0 & a \\
b & 0 & 0 \\
0 & c & 0
\end{array}\right) .
$$

It is routine to check that the twelve elements of $H$ form a group under multiplication and that $H \cong A_{4}$. 
Let $R$ be the subring of $S$ generated by $H$. We shall use $e_{i j}$ to denote the $3 \times 3$ matrix with 1 in the $(i, j)$-position and 0's elsewhere. By taking sums of pairs of elements of $H$ it is easy to show that $2 e_{i j} \in R$ for all $i$ and $j$. Thus $2 S \subseteq R$. We shall identify $S / 2 S$ with $M_{3}(F)$ where $F=\mathbb{Z} / 2 \mathbb{Z}$. Then $R / 2 S$ is the group algebra $F\left[C_{3}\right]$ where $C_{3}$ is the cyclic group of order 3 generated by the element $e_{12}+e_{23}+e_{31}$ of $M_{3}(F)$. Thus $R$ is the subring of $S$ such that $2 S \subseteq R$ (from which it follows that $R$ is a prime ring) and $R / 2 S=F\left[C_{3}\right]$, and $R / 2 S$ is the direct sum of a field with 2 elements and a field with 4 elements. In order to match the notation used in [1] we set $T=2 S$. Then $T$ is the intersection of those maximal ideals of $R$ which contain the ideal $2 S$ of $S$, and $\lambda(R / T)=2$ where $\lambda$ denotes length as an $R / T$-module. Set

$$
T^{*}=\left\{w \in M_{3}(\mathbb{Q}): w T \subseteq R\right\} .
$$

Clearly $S \subseteq T^{*}$ and $S / R$ has $2^{6}$ elements. Also $S / R$ is an $R / T$-module where $R / T$ is the direct sum of two fields as above, from which it follows that $\lambda(S / R) \geq 3$. Therefore $\lambda\left(T^{*} / R\right) \neq \lambda(R / T)$, so that $R$ does not have injective dimension 1 [1, Theorem 5.7]. But $R \cong \mathbb{Z}\left[A_{4}\right] / P$ for some minimal prime ideal $P$ of $\mathbb{Z}\left[A_{4}\right]$.

3. The condition. Throughout this section $R$ will denote a semi-prime Noetherian ring of injective dimension 1 with quotient ring $Q$, and $I$ and $J$ will denote ideals of $R$ such that each is the annihilator of the other (note that if $U$ and $V$ are ideals of $R$ then, because $R$ is semi-prime, we have $U V=0$ if and only if $V U=0$ if and only if $U \cap V=0$ ). We need to establish some general properties of $R, I$, and $J$ before we can prove the necessary and sufficient condition for $R / P$ to have injective dimension 1 where $P$ is a minimal prime ideal of $R$.

Notation 3.1. For a subset $X$ of $Q$ set

$$
X^{*}=\{q \in Q: q X \subseteq R\}
$$

and

$$
X_{*}=\{q \in Q: X q \subseteq R\} .
$$

The following result will be needed; it is an easy consequence of a result in [2] and we shall only give it in the form which we need.

THEOREM 3.2. (Jans) Let $W$ be a finitely-generated right $R$-submodule of $Q$ such that if $q \in Q$ with $q W=0$ then $q=0$. Then $W=\left(W^{*}\right)_{*}$.

Proof. Because $W_{R}$ is finitely generated, there is a regular element $c$ of $R$ such that $c W \subseteq R$. Thus $W_{R}$ embeds in $R_{R}$, so that $W_{R}$ is torsionless. Also it follows from the other assumption made on $W$ that we can identify $W^{*}$ with $\operatorname{Hom}\left(W_{R}, R_{R}\right)$ and $\left(W^{*}\right)_{*}$ with $\operatorname{Hom}\left({ }_{R}\left(W^{*}\right),{ }_{R} R\right)$. Because $W_{R}$ is torsionless and $R$ has injective dimension 1 , it follows from [2, Corollary, p. 72] that $W_{R}$ is reflexive and hence $W=\left(W^{*}\right)_{*}$.

We shall now study $R, I$, and $J$ as defined in the first paragraph of this section.

Proposition 3.3. We have $J^{*}=J_{*}=I Q+R=\{q \in Q: q J \subseteq J\}=\{q \in Q: J q \subseteq J\}$, and similarly with I and $J$ interchanged.

Proof. There are central idempotents $e$ and $f$ of $Q$ such that $e+f=1$, $e Q=I Q=Q I$, and $f Q=J Q=Q J$. We shall first show that $(I+J)^{*}=e R+f R$. 
Set $H=e R+f R$. Clearly $R \subseteq H$. By 3.2 we have $H=\left(H^{*}\right)_{*}$. Let $w \in H^{*}$. Then $e w=$ $w e \in R$ and $e w J=0$, so that $e w \in I$. Similarly $f w \in J$, and $w=e w+f w \in I+J$. Thus $H^{*} \subseteq I+J$. On the other hand $I H=I e R+I f R$ with $I f=0$. Hence $I H=I e R=$ $I e=I$, so that $I \subseteq H^{*}$. Similarly $J \subseteq H^{*}$, and it follows that $I+J=H^{*}$. Therefore $H=\left(H^{*}\right)_{*}=(I+J)_{*}$. But, because $e$ and $f$ are central, we have $H^{*}=H_{*}=\{q \in Q$ : $q e \in R$ and $q f \in R\}$, so that by symmetry we also have $H=\left(H_{*}\right)^{*}=(I+J)^{*}$.

Next we shall show that $J^{*}=I Q+R$. We have $I Q J=0$, so that $I Q+R \subseteq J^{*}$. Let $x \in J^{*}$. Then $x=x e+x f$ with $x e J=0$. Hence $x e \in J^{*}$ and so also $x f=x-x e \in J^{*}$. We have $x f I=0$ so that $x f \in I^{*}$. Thus $x f \in I^{*} \cap J^{*}=(I+J)^{*}=H=e R+f R$, from which it follows that $x f \in f R$. Therefore $x=x e+x f \in e Q+f R=e Q+R=I Q+$ $R$, which completes the proof that $J^{*}=I Q+R$.

By symmetry we also have $J_{*}=I Q+R$. Also $J^{*} J I=0$ with $J^{*} J \subseteq R$, so that $J^{*} J \subseteq J$. Therefore $J^{*}=\{q \in Q: q J \subseteq J\}$.

Proposition 3.4. The injective dimension of I as an $R / J$-module is 1.

Proof. By symmetry, it does not matter whether we work on the right or the left. Because $Q / R$ is $R$-injective it follows that $J^{*} / R$ (being the annihilator of $J$ in $Q / R$ ) is $R / J$-injective. But by 3.3 we have $J^{*} / R=(I Q+R) / R \cong I Q /(I Q \cap R)=I Q / I$. Also $Q$ is $R$-injective so that $I Q$ (being the annihilator of $J$ in $Q$ ) is $R / J$-injective. Thus both $I Q$ and $I Q / I$ are injective as $R / J$-modules. We note also that $I Q$ is the injective envelope of $I$ as an $R / J$-module.

Proposition 3.5. The ring $R /(I+J)$ is $Q F$.

Proof. Set $K=I+J$ and $H=e R+f R$ as in the proof of 3.3 (where it was shown that $H=K^{*}$ ). Thus $H / R$ is the annihilator of $K$ in $Q / R$. Because $R$ has injective dimension 1 we know that $Q / R$ is $R$-injective and hence $H / R$ is $R / K$-injective. We shall prove that $R / K$ is a self-injective ring by showing that $R / K \cong H / R$ as right $R$-modules. Define $a: R \rightarrow H / R$ by $a(x)=e x+R$ for all $x \in R$. Then $a$ is a right $R$-module homomorphism, and $a$ is surjective because $e R+R=e R+f R=H$. It is now enough to show that $\operatorname{Ker}(a)=K$. Let $x \in R$. The following statements are equivalent: $e x \in R$; ex $\in R$ and $f x \in R$ (because $f x=x-e x$ ); ex $\in I$ and $f x \in J$; $x \in I+J$. Therefore $a(x)=0$ if and only if $x \in K$.

We next study the quotient ring of $R / J$, and we find it necessary to be very careful as follows about the notation to be used.

Notation 3.6. Let ' denote image in the semi-simple ring $Q^{\prime}=Q / f Q$. Then $R^{\prime}=(R+f Q) / f Q \cong R /(R \cap f Q)=R / J$. Thus we can identify $R / J$ with $R^{\prime}$, and the quotient ring of $R^{\prime}$ is $Q^{\prime}$. Also $I^{\prime}=(I+f Q) / f Q \cong I /(I \cap f Q) \cong I$ because $I \cap f Q=0$. Similarly $I \cong(I+J) / J$. Therefore it follows from 3.4 that $I^{\prime}$ has injective dimension 1 as an $R^{\prime}$-module, and from 3.5 that $R^{\prime} / I^{\prime}$ is a $\mathrm{QF}$ ring.

Proposition 3.7. With the notation of 3.6 set

$$
W=\left\{w \in Q^{\prime}: w I^{\prime} \subseteq I^{\prime}\right\}
$$

Then $W=R^{\prime}$.

Proof. Clearly $R^{\prime} \subseteq W$. Let $w \in W$. We have $w=q^{\prime}$ for some $q \in Q$. Because $w I^{\prime} \subseteq I^{\prime}$ we have $(q I)^{\prime} \subseteq I^{\prime}$, i.e. $q I \subseteq I+f Q$. But $I \subseteq e Q$ where $e$ is central and $e f=0$. Therefore $q I \subseteq I$. Hence by 3.3 we have $q \in J Q+R=R+f Q$, so that $w=q^{\prime} \in R^{\prime}$. 
COROLlary 3.8. Let $x \in Q^{\prime}$. Then $x I^{\prime} \subseteq R^{\prime}$ if and only if $I^{\prime} x \subseteq R^{\prime}$.

Proof. Suppose that $x I^{\prime} \subseteq R^{\prime}$. Then $I^{\prime} x I^{\prime} \subseteq I^{\prime}$, so that $I^{\prime} x \subseteq R^{\prime}$ by 3.7.

Proposition 3.9. The $R^{\prime}$-module $Q^{\prime} / I^{\prime}$ is injective.

Proof. This is because $Q^{\prime}$ is the injective envelope of $I^{\prime}$, and $I^{\prime}$ has injective dimension 1 as explained in 3.6.

We can now prove our main result.

THEOREM 3.10. The ring $R / J$ has injective dimension 1 if and only if $(I+J) / J$ is an invertible ideal of $R / J$.

Proof. With the notation of 3.6 we need to show that $R^{\prime}$ has injective dimension 1 if and only if $I^{\prime}$ is an invertible ideal of $R^{\prime}$. Suppose that $R^{\prime}$ has injective dimension 1. By 3.5 we know that $R^{\prime} / I^{\prime}$ is a QF ring. Set $W=\left\{w \in Q^{\prime}: w I^{\prime} \subseteq R^{\prime}\right\}$ and note that by 3.8 we also have $W=\left\{w \in Q^{\prime}: I^{\prime} w \subseteq R^{\prime}\right\}$. Then $W I^{\prime}$ is an ideal of $R^{\prime}$ with $I^{\prime} \subseteq W I^{\prime}$. Let $x \in R^{\prime}$ with $x W I^{\prime} \subseteq I^{\prime}$. Then $x W \subseteq R^{\prime}$ by 3.7. Adapting the notation of 3.1 to $R^{\prime}$ and $Q^{\prime}$ rather than $R$ and $Q$, we have $W=\left(I^{\prime}\right)^{*}=\left(I^{\prime}\right)_{*}$ and $x \in W^{*}$. But $R^{\prime}$ has injective dimension 1 , so that $W^{*}=\left(\left(I^{\prime}\right)_{*}\right)^{*}=I^{\prime}$ by 3.2. Therefore $x \in I^{\prime}$. Thus the ideal $W I^{\prime} / I^{\prime}$ of the QF ring $R^{\prime} / I^{\prime}$ has zero left annihilator, so that $W I^{\prime} / I^{\prime}=R^{\prime} / I^{\prime}$, i.e. $W I^{\prime}=R^{\prime}$. By symmetry we also have $I^{\prime} W=R^{\prime}$, so that $I^{\prime}$ is an invertible ideal of $R^{\prime}$.

Conversely suppose that $I^{\prime}$ is an invertible ideal of $R^{\prime}$. By 3.9 we know that $Q^{\prime} / I^{\prime}$ is an injective $R^{\prime}$-module. It follows from 3.12 (below) that $Q^{\prime} / R^{\prime}$ is injective, and so $R^{\prime}$ has injective dimension 1 .

COROLlARY 3.11. Let $R$ be a semi-prime Noetherian ring of injective dimension 1 , and let $J$ be a minimal prime ideal of $R$. Let $I$ be the intersection of all the minimal prime ideals of $R$ other than $J$ (with $I=R$ if there are none). Then the ring $R / J$ has injective dimension 1 if and only if $(I+J) / J$ is an invertible ideal of $R / J$.

LEMMA 3.12. Let $R$ be any ring with an ideal I which is invertible in some over-ring $Q$ of $R$. Then $Q / R$ is a direct summand of the direct sum of a finite number of copies of $Q / I$ (as right $R$-modules).

Proof. We can fix $x_{1}, \ldots, x_{n} \in I$ and $w_{1}, \ldots, w_{n} \in Q$ such that $w_{1} x_{1}+\ldots+$ $w_{n} x_{n}=1$ and $w_{i} I \subseteq R$ for all $i$. Set $X=(Q / I)^{(n)}$ and define $a: Q / R \rightarrow X$ by

$$
a(q+R)=\left(x_{1} q+I, \ldots, x_{n} q+I\right)
$$

for all $q \in Q$. Then $a$ is a right $R$-module homomorphism. Define $b: X \rightarrow Q / R$ by

$$
b\left(\left(q_{1}+I, \ldots, q_{n}+I\right)\right)=w_{1} q_{1}+\cdots+w_{n} q_{n}+R
$$

for all $q_{i} \in Q$. It is easy to check that $b a(q+R)=q+R$ for all $q \in Q$, so that $a$ splits and $Q / R$ is isomorphic to a direct summand of $X$.

\section{REFERENCES}

1. A. W. Chatters and C. R. Hajarnavis, Noetherian rings of injective dimension one which are orders in quasi-Frobenius rings, J. Algebra 270 (2003), 249-260.

2. J. P. Jans, Rings and homology (Holt, Rinehart and Winston, New York, 1964).

3. L. S. Levy, Torsion-free and divisible modules over non-integral domains, Canadian J. Math. 15 (1963), 132-151. 